Gut, 1981, 22, 301-305

\title{
Impaired glucose tolerance and growth hormone in chronic liver disease
}

\author{
W J RILEY* AND V J MCCANN \\ From the Department of Biochemistry and Diabetic Unit, Royal Perth Hospital, Perth, Western Australia
}

SUMMARY Of 30 patients with chronic liver disease 16 showed some degree of impairment of glucose tolerance, and 16 patients had lack of suppression of raised fasting growth hormone levels or showed an anomalous rise after oral glucose. No relationship, however, existed between the state of glucose tolerance and the presence of abnormal growth hormone levels. Plasma glucose in those with normal growth hormone response at $0, \frac{1}{2}, 1,1 \frac{1}{2}$, and 2 hours, after $50 \mathrm{~g}$ glucose were $5 \cdot 55 \pm$ $0.41 \mathrm{mmol} / \mathrm{l}, 8 \cdot 71 \pm 0 \cdot 59,10 \cdot 66 \pm 0.99,10 \cdot 28 \pm 1 \cdot 37,8.90 \pm 1 \cdot 40($ mean $\pm \mathrm{SEM} ; \mathrm{n}=14)$. Under the same conditions those with abnormal growth hormone responses showed values of $5 \cdot 32 \pm 0 \cdot 59$, $7 \cdot 83 \pm 0 \cdot 81,9 \cdot 41 \pm 0 \cdot 95,9 \cdot 46 \pm 0 \cdot 99,8 \cdot 69 \pm 0 \cdot 98$. At no time were the differences significantly different as judged by Student's $t$ test. Measurement of serum insulin indicated a relative deficiency in patients with impaired tolerance. It is concluded that the abnormal growth hormone is not directly responsible for the impaired glucose tolerance.

Several authors ${ }^{1-6}$ have described increased levels of growth hormone in peripheral plasma in patients with chronic liver disease. These studies have indicated both an increase in fasting values and lack of suppression or even a paradoxical rise in plasma levels after glucose loading.

The cause of the raised growth hormone levels in patients with liver disease remains uncertain and has been attributed to impaired degradation ${ }^{7}$ or increased secretion. ${ }^{8}$

Although the implications of the raised growth hormone levels in the pathogenesis of the glucose intolerance which develops in such patients has been discussed, no firm conclusions can be drawn in regard to its role in this situation. Only one study has attempted to correlate glucose intolerance in individual patients with abnormality in growth hormone levels. These authors showed that in a group of patients with chronic hepatic cirrhosis those with impaired glucose tolerance showed higher growth hormone levels during the GTT than those cirrhotic patients with normal glucose tolerance. They concluded, therefore, that high growth

\footnotetext{
*Address for correspondence: Dr W J Riley, Department of Biochemistry, Royal Perth Hospital, GPO Box X2213, Perth, Western Australia, 6001.

Reprints will not be available from the authors

Received for publication 29 October 1980
}

hormone levels may be responsible for the high incidence of abnormal glucose tolerance in hepatic cirrhosis.

The present work describes a similar study in a group of patients with chronic liver disease in whom we investigated the correlation between the degree of glucose tolerance and the level of circulating growth hormone.

\section{Methods}

\section{PATIENTS}

Plasma glucose, insulin, and growth hormone levels were estimated during an oral glucose tolerance test in 30 patients with chronic liver disease. These patients were selected on the basis of the usual clinical features of hepatic cirrhosis: enlarged liver and spleen, palmar erythema, spider naevi, and signs of portal hypertension, together with the laboratory findings of hyperbilirubinaemia, hypoalbuminaemia, hypergammaglobulinaemia, and a rise in transaminases. In the majority the diagnosis was confirmed by liver biopsy. Sixteen patients had alcoholic cirrhosis, eight had active chronic hepatitis, five had cryptogenic cirrhosis, and one had biliary cirrhosis.

Patients were classified as 'diabetic', 'impaired glucose tolerance', or 'normal' with respect to a $50 \mathrm{~g}$ oral GTT according to the criteria shown in Table 1. 
Table 1 Criteria for classification of patients based on plasma glucose levels during oral GTT

\begin{tabular}{lccc}
\hline & \multicolumn{2}{l}{ Plasma glucose $($ mmol $/ \mathrm{l})$} \\
\cline { 2 - 3 } & Normal & Impaired & Diabetic \\
Fasting & $<6.0$ & & $>8.0$ \\
1 hour & $<10.0$ & $10.1-13.9$ & $>14.0$ \\
2 hour & $<8.0$ & $8.1-10.9$ & $>11.0$ \\
\hline
\end{tabular}

ANALYTICAL TECHNIQUES

Plasma glucose was measured with a Beckman glucose analyser or a Technicon autoanalyser using a glucose oxidase method.

Plasma insulin and growth hormone were measured by radioimmunoassay using a double antibody technique based on the method described by Morgan and Lazarow. ${ }^{9}$ The standard used for insulin assays was the WHO International Reference Preparation of Insulin, Human, for Immunoassay, For growth hormone assays, the Australian HGH radioimmunoassay standard (no. 62.4 (50) F $8 \mathrm{R}$ ) distributed by the Human Pituitary Advisory Committee of the Australian Government has been used: $1 \mathrm{ng}$ of this material is immunologically equivalent to $2 \mu \mathrm{U}$ of the WHO 1st International Reference Preparation for Immunoassay, and results are reported in terms of this latter material.

\section{Results}

Of the 30 patients examined 16 were classified as having normal glucose tolerance (group A), 19 with having impaired glucose tolerance (group B), and four being diabetic (group C). The mean glucose levels for the groups during the glucose tolerance test are shown in Fig. 1. Impaired glucose tolerance was not confined to any one aetiological group and occurred in four patients with alcoholic cirrhosis, three with active chronic hepatitis, two with cryptogenic cirrhosis and one with biliary cirrhosis.

The insulin responses in the three groups are shown in Fig. 2. Although the two groups having impaired tolerance to glucose appear also to have impaired insulin production, the variation in individual responses was so great that no statistically significant difference between any groups can be claimed for any sampling time. If one takes, however, the area under the curve and above the baseline fasting level as a measure of the total amount of insulin secreted in response to a glucose challenge, group A patients show a greater response than combined group B and C. The difference is statistically significant $(t=2 \cdot 60, \mathrm{P}=<0 \cdot 02)$.

Group A patients showed a mean response which was not different from the reference range quoted in

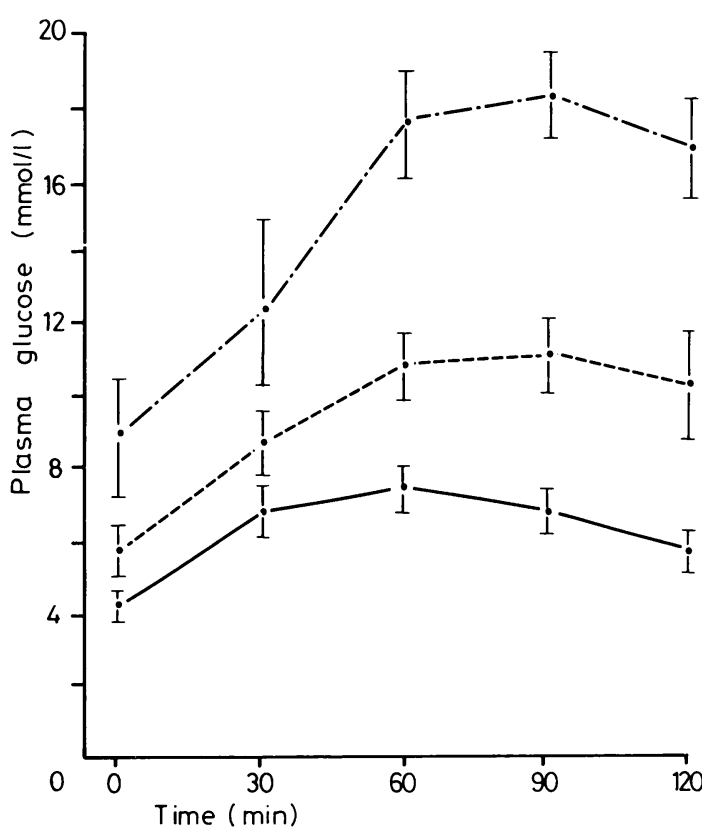

Fig. 1 Plasma glucose levels during oral GTT. Means and SEM are shown for each group classified according to Table 1. — group A normal, - - - group B impaired ...... group $C$ diabetic.

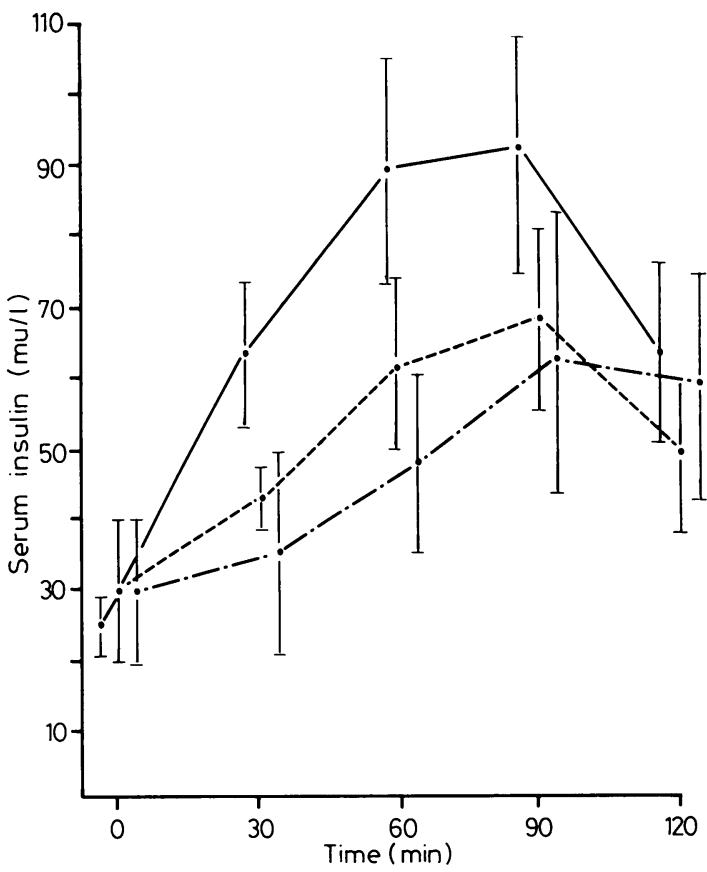

Fig. 2. Serum insulin levels (mean and SEM) during oral GTT for groups classified according to Table 1 .

- normal glucose response, - - - - impaired glucose response -._._. diabetic glucose response. 


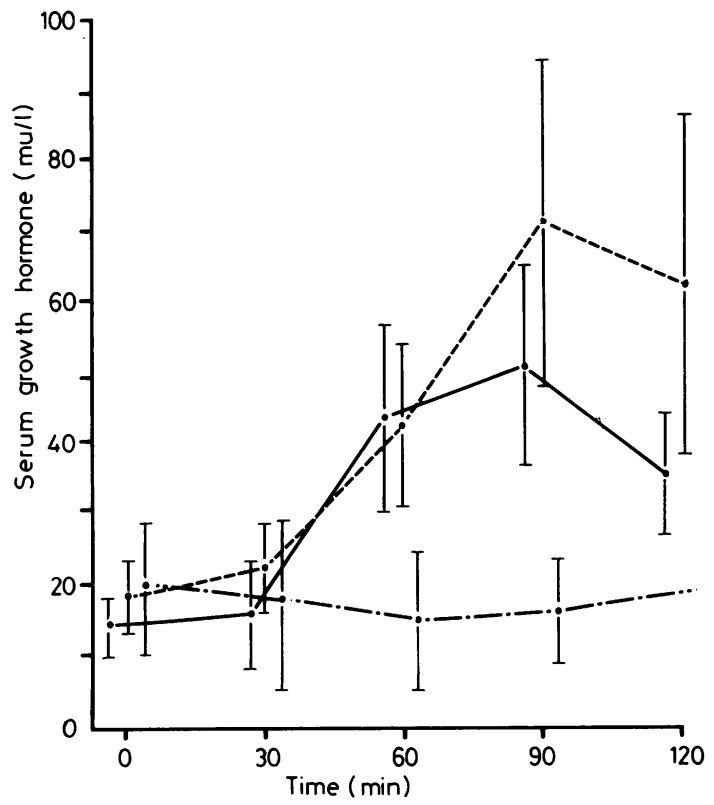

Fig. 3 Serum growth hormone levels (mean and SEM) during oral GTT for groups classified according to Table 1. $\longrightarrow$ normal, ...- - impaired, -..... diabetic.

this laboratory (fasting: $3-26 \mathrm{mU} / \mathrm{L}, 30$ minutes: 15-125 mU/L, 60 minutes: $13-131 \mathrm{mU} / \mathrm{L}, \quad 120$ minutes: $6-60 \mathrm{mU} / \mathrm{L}$.

Those patients with impaired and diabetic GTT all showed insulin responses which fell within the reference range, so that insulin levels are inadequate with respect to the level of blood glucose.

Growth hormone responses in the three groups are shown in Fig. 3. Again no significant difference occurs between any group at any sampling time. As compared with the reference range used in this laboratory, the fasting levels are raised to a similar degree in all groups. Reference values were previously established on 100 fasting, resting, healthy volunteers (mean value $8 \mathrm{mU} / \mathrm{L}$, SEM $0.8 \mathrm{mU} / \mathrm{L}$ ). This group was not age and sex matched for the patient's under study. Both group A and group B exhibit clearly abnormal growth hormone responses to glucose loading with an increase occurring within 60 minutes. Group $C$ appears to have a more normal response, but the numbers in this group are very small and there is marked variation between individuals.

One can view the same data in a slightly different light by classifying patients according to the growth hormone response exhibited and examining the glucose tolerance in each group. Thus patients were classified as normal or abnormal growth hormone responders, as shown in Table 2. Although, for convenience, the terms normal and abnormal have been used, it makes no difference to our subsequent argument if the two groups are regarded simply as having different degrees of impairment.

Comparison of the insulin response in those patients with normal or abnormal growth hormone response is shown in Fig. 4. Again the two groups show similar circulating insulin levels at all sampling points. The data do not provide any evidence to suggest that increased growth hormone levels are associated with increased insulin output to maintain similar glucose levels in the two groups.

Table 2 Growth hormone values in groups regarded as having normal and abnormal growth hormone responses

\begin{tabular}{cll}
\hline Time after glucose (min) & \multicolumn{2}{l}{ Growth hormone* $(\mathrm{mU} / \mathrm{l})$} \\
\cline { 2 - 3 } & Normal $(10 \mathrm{pts})$ & Abnormal $(20 \mathrm{pts})$ \\
\hline 0 & $7 \cdot 8 \pm 2 \cdot 3$ & $20 \cdot 7 \pm 3 \cdot 1$ \\
30 & $4 \cdot 4 \pm 0 \cdot 3$ & $26 \cdot 1 \pm 5 \cdot 5$ \\
60 & $5 \cdot 2 \pm 0 \cdot 7$ & $56 \cdot 4 \pm 10 \cdot 2$ \\
90 & $6 \cdot 1 \pm 1 \cdot 1$ & $77 \cdot 2 \pm 13 \cdot 0$ \\
120 & $4 \cdot 7 \pm 0 \cdot 8$ & $62 \cdot 8 \pm 12 \cdot 8$ \\
\hline
\end{tabular}

*Mean \pm SEM.

Patients were classified as normal if growth hormone levels satisfied one of the following criteria: (1) All values $<10$ (six patients). (2) No value $>14$ Mean value $<8$ (three patients). (3) Fasting value $>14<30$ All other values $<5 \mathrm{mU} / \mathrm{l}$ (one patient).

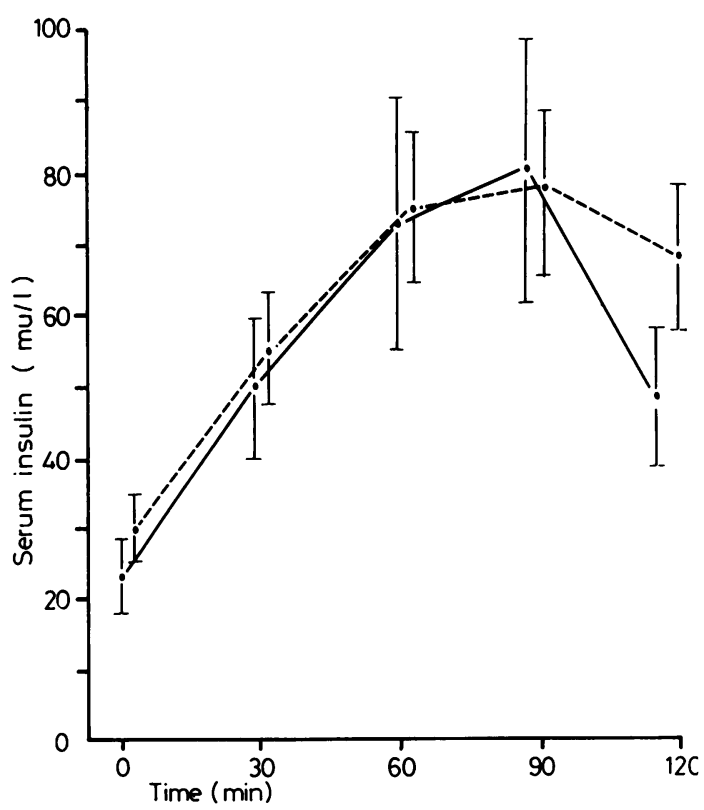

Fig. 4 Serum insulin levels (mean $\pm S E M$ ) during oral GTT in those patients showing normal a and raised - - - growth hormone levels. 


\section{Discussion}

The occurrence of impaired glucose tolerance ${ }^{10}$ and a rise in peripheral growth hormone levels in chronic liver disease ${ }^{1-6}$ have been well described. The findings of this study in this respect are in agreement with published findings. Thus, approximately $20-50 \%$ of cirrhotic patients are expected to show some degree of impairment of glucose tolerance and in our group 14 out of 30 were so classified. Similarly, we found that a very high proportion of our patients showed a paradoxical lack of suppression or even a rise in growth hormone levels after a $50 \mathrm{~g}$ glucose challenge. Sixteen of our 30 patients were classified as abnormal in this respect. In addition, four patients had moderately increased fasting levels of growth hormone but showed normal suppression after glucose.

Although the abnormalities in glucose tolerance and growth hormone levels are well documented, the relationship between the two is not clear. Samaan and co-workers ${ }^{3}$ found that abnormalities in growth hormone were more marked in those patients with hepatic cirrhosis who had impaired glucose tolerance than in those with normal glucose tolerance. They suggested, therefore, that the high levels of growth hormone and their lack of suppression with hyperglycaemia may play a role in producing an insensitivity to insulin in patients with liver disease.

Our findings are in disagreement with this, in that no statistical significance can be found with respect to degree of growth hormone abnormality and degree of glucose intolerance. No satisfactory explanation is readily available to explain this difference in findings, but, of course, it is possible that our patient population was in some way different from that of Samaan and his colleagues. It is also possible that it may be due to the very wide differences in growth hormone response seen in individual patients in our series. In an attempt to overcome this, patients were classified according to whether they had a normal or abnormal growth hormone response to glucose. As demonstrated in Fig. 5 no difference in glucose tolerance could be discerned in the two groups; and it therefore appears doubtful as to whether growth hormone plays a significant role in producing abnormalities in the handling of glucose in these patients.

If the raised growth hormone levels were responsible for promoting hyperinsulinism and insulin resistance in patients with liver disease one might expect to find differences in the insulin response to glucose in the group with abnormal growth hormone response as compared with that with normal growth hormone. That this is not so is seen in Fig. 4 and

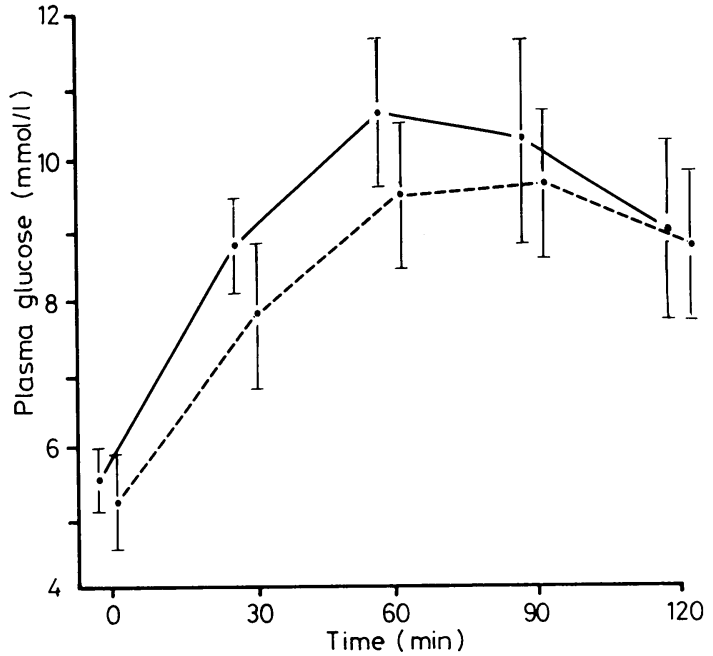

Fig. 5 Plasma glucose levels (mean $\pm S E M$ ) during oral GTT in those patients showing normal (__ raised (- - -) growth hormone levels.

again we have failed to produce any evidence that growth hormone plays a role in this regard.

Previous studies ${ }^{310}$ have, in fact, indicated that hyperinsulinism is a common finding in liver disease. Moreover, the studies of Samaan and co-workers have indicated that the exaggerated insulin response to glucose is associated with those patients exhibiting a normal tolerance for glucose. Where there is impaired glucose tolerance the insulin response is lessened. These findings are confirmed by the present work, which indicates that a smaller increment of insulin over the fasting level occurs in these patients as compared with those with normal glucose tolerance.

In conclusion, the group of patients we have studied appear to be similar to those observed by other workers both in respect to the number exhibiting impaired glucose tolerance, in the nature of their insulin response to glucose, and in respect to abnormal circulating growth hormone levels. We have not, however, been able to demonstrate any relationship between the latter and the degree of impairment of glucose tolerance or the nature of the insulin response. It would seem unlikely from our results that growth hormone plays a major role in the development of impaired glucose tolerance. Other workers have commented on the possibility that oestrogens may inhibit peripheral effects of growth hormone in these patients, as they do not develop clinical features such as those seen in acromegaly. It is possible that the effects of growth hormone on carbohydrate metabolism are also inhibited by some unidentified factor. 
The reduced insulin secretion in patients with impaired glucose tolerance would suggest that this is related to impaired beta cell function rather than to insulin resistance. There are a number of ways in which the association may occur-for example, patients with alcoholic liver disease may well have an associated alcoholic pancreatitis and it has been shown that both active chronic hepatitis ${ }^{11}$ and juvenile onset diabetes ${ }^{12} 13$ share an association with the histocompatibility antigen HLA B8.

Additional investigation is required to clarify the cause of impaired glucose tolerance in patients with chronic liver disease but it seems likely that the association arises through more than one mechanism.

\section{References}

${ }^{1}$ Becker MD, Cook GC, Wright AD. Paradoxical elevation of growth hormone in active chronic hepatitis. Lancet $1969 ; 2$ : 1035-8.

${ }^{2}$ Hernandez A, Zorilla E, Gerghberg H. Decreased insulin production, elevated growth hormone levels and glucose intolerance in liver disease. J Lab Clin Med 1969; 75: 25-33.

${ }^{3}$ Samaan NA, Stone DB, Eckhardt RD. Serum glucose, insulin and growth hormone in chronic hepatic cirrhosis. Arch Intern Med 1969; 124: 149-52.

${ }^{4}$ Conn HO, Daughaday WH. Cirrhosis and diabetes V.
Serum human growth hormone levels in Laennec's cirrhosis. J Lab Clin Med 1970; 76: 678-88.

${ }^{5}$ Greco AV, Ghirlanda G, Patrono C, Fedeli G, Manna R. Behaviour of pancreatic glucagon, insulin, and $\mathrm{HGH}$ in liver cirrhosis, after arginine and IV glucose. Acta Diabetol Lat 1974; 11 : 330-9.

${ }^{6}$ Zanoboni A, Zanoboni-Muciaccia W. Elevated basal growth hormone levels and growth hormone response to TRH in alcoholic patients with cirrhosis. $J$ Clin Endocrinol Metab 1977; 45: 576-8.

${ }^{7}$ Cameron DP, Burger HG, Catt KJ, Gordon E, Watts JMcK. Metabolic clearance of human growth hormone in patients with hepatic and renal failure, and in the isolated perfused pig liver. Metabolism 1972; 21 : 895-904.

${ }^{8}$ Taylor AL, Lipman RL, Salam A, Mintz DH. Hepatic clearance of human growth hormone. J Clin Endocrinol Metab 1972; 34: 395-9.

${ }^{9}$ Morgan CR, Lazarow A. Immunoassay of insulin: two antibody system. Diabetes 1963: 12: 115-26.

${ }^{10}$ Megyesi C, Samols E, Marks V. Glucose tolerance and diabetes in chronic liver disease. Lancet 1967; 2: 1051-5.

${ }^{11}$ Galbraith RM, Eddleston ALWF, Smith MGM, Wellcams R, et al. Histocompatibility antigens in active chronic hepatitis and primary biliary cirrhosis. $\mathrm{Br} \mathrm{Med}$ $J$ 1974; 3: 604-5.

${ }^{12}$ Nerup J, Platz P, Andersen OO, Christy M, et al. HL-A antigens and diabetes mellitus. Lancet 1974; 2: 864-6.

${ }^{13}$ Cudworth AG, Woodrow JC. HL-A system and diabetes mellitus. Diabetes 1975; 24: 345-9. 